\title{
On the impact of model selection on predictor identification and parameter inference
}

\author{
Ruth M. Pfeiffer ${ }^{1}$ • Andrew Redd ${ }^{2}$. \\ Raymond J. Carroll ${ }^{3}$
}

Received: 6 March 2015 / Accepted: 24 September 2016 / Published online: 22 October 2016 (C) The Author(s) 2016. This article is published with open access at Springerlink.com

\begin{abstract}
We assessed the ability of several penalized regression methods for linear and logistic models to identify outcome-associated predictors and the impact of predictor selection on parameter inference for practical sample sizes. We studied effect estimates obtained directly from penalized methods (Algorithm 1), or by refitting selected predictors with standard regression (Algorithm 2). For linear models, penalized linear regression, elastic net, smoothly clipped absolute deviation (SCAD), least angle regression and LASSO had a low false negative (FN) predictor selection rates but false positive (FP) rates above $20 \%$ for all sample and effect sizes. Partial least squares regression had few FPs but many FNs. Only relaxo had low FP and FN rates. For logistic models, LASSO and penalized logistic regression had many FPs and few FNs for all sample and effect sizes. SCAD and adaptive logistic regression had low or moderate FP rates but many FNs. 95\% confidence interval coverage of predictors with null effects was approximately $100 \%$ for Algorithm 1 for all methods, and $95 \%$ for Algorithm 2 for large sample and effect sizes. Coverage was low only for penalized partial least squares (linear regression). For outcome-associated predictors, coverage was close to $95 \%$ for Algorithm 2 for large sample and effect sizes for all methods except penalized partial least squares and penalized logistic regression. Coverage
\end{abstract}

Electronic supplementary material The online version of this article (doi:10.1007/s00180-016-0690-2) contains supplementary material, which is available to authorized users.

Ruth M. Pfeiffer

pfeiffer@mail.nih.gov

1 Division of Cancer Epidemiology and Genetics, National Cancer Institute, 9609 Medical Center Drive, Room 7E142, Bethesda, MD 20892, USA

2 Department of Internal Medicine, University of Utah School of Medicine, Salt Lake City, UT 84132, USA

3 Department of Statistics, Texas A\&M University, College Station, TX 77843-3143, USA 
was sub-nominal for Algorithm 1. In conclusion, many methods performed comparably, and while Algorithm 2 is preferred to Algorithm 1 for estimation, it yields valid inference only for large effect and sample sizes.

Keywords Biased estimates - Post-model selection inference · Finite sample inference $\cdot$ Shrinkage $\cdot$ Variable selection

\section{Introduction}

Many regression procedures have been proposed in the recent literature that use penalties on regression coefficients in order to achieve sparseness or shrink them toward zero. These methods are popular for the analysis of datasets with large numbers of predictors, as they allow efficient selection of regression variables. While in many applications the primary interest is in identifying outcome associated covariates, it is nonetheless sometimes also desirable to gain scientific insights into the data generating process, and draw statistical inference on the parameters associated with the selected variables. Biases in estimates as well as in standard errors and confidence intervals become important if investigators focus on the magnitude of the observed effects.

Selection of predictor variables is a special case of model selection, which can be stated as follows. Let $\mathcal{M}$ denote the space of all candidate models that could be used to describe the data $D$. For our purposes $\mathcal{M}$ is characterized in terms of distribution functions that depend on parameters $\boldsymbol{\beta}$ and may or may not contain the true model that gave rise to the data. The model selection problem is to choose a model $\hat{M}(D)$ in $\mathcal{M}$ such that $\hat{M}$ is a "good" model in terms of parameter estimation or prediction. If the focus is on inference regarding the parameters, then the quantity of interest is $\hat{\boldsymbol{\beta}}(\hat{M})$. Model selection is a source of variability that is often ignored in standard statistical approaches. However, several authors, e.g. Sen (1979), Pötscher (1991) and Leeb (2005), have shown that the asymptotic distribution of the post-model selection estimates $n^{1 / 2}(\hat{\boldsymbol{\beta}}-\boldsymbol{\beta})$, where $n$ denotes the sample size, is typically non-normal, and depends on the unknown $\boldsymbol{\beta}$ in complex fashions.

Some analytical results are available for penalized maximum likelihood estimators obtained from LASSO (Tibshirani 1996), SCAD (smoothly clipped absolute deviation; Fan and Li 2001) and hard thresholding for linear regression models, see e.g. Knight and Fu (2000), Leeb and Pötscher (2009) and Pötscher and Schneider (2009). These estimators have highly non-normal finite sample distributions and under conservative model selection their large sample distribution can be far from normal. Even under consistent model selection (pointwise) asymptotic analysis gives highly misleading results. In addition, the large sample properties depend on the choice of tuning parameters. Therefore the naively estimated standard error for those estimates will be biased and confidence intervals based on standard asymptotic theory for these methods may not have proper coverage, not even asymptotically.

No comprehensive comparisons of penalized approaches with respect to their finite sample properties have been performed to date, and little work has been done for non-linear models. We thus studied the properties of estimates obtained from popular penalized likelihood approaches applied to linear and logistic regression models 
using simulated data, focusing on realistic effect and sample sizes to make conclusions applicable to practical settings (Sect. 2.2). We first assess the methods' ability to identify truly outcome-associated predictors. We then study properties of effect estimates obtained directly from penalized methods (Algorithm 1), or by refitting selected predictors with standard regression (Algorithm 2) described in Sect. 2.3. The results presented in Sect. 3 can help to avoid overly optimistic interpretation of parameters in future research.

\section{Methods}

The goal of this analysis is to assess the impact of model selection on parameter estimates in linear and logistic models. We evaluated the influence of sample size, $n$, and magnitude of the regression coefficients for associations $\boldsymbol{\beta}$ on each method's ability to identify outcome associated predictors. We also studied properties of effect estimates obtained directly from penalized methods (Algorithm 1), or by refitting selected predictors with standard regression (Algorithm 2). A range of sample sizes, algorithms and correlation structures among predictors are utilized.

\subsection{Estimation methods and algorithms}

We calculated both the LASSO (Tibshirani 1996), and least angle regression (LARS, Efron et al. 2004) estimates with the function lars in the lars library of the statistical package $\mathrm{R}$ (Ihaka and Gentlemen 1996). The elastic net was fit using the function enet in the elasticnet library (Zou and Hastie 2005). We used the function relaxo in the library relaxo in R to fit relaxed LASSO, a generalization of the LASSO shrinkage technique (Meinshausen 2007). Generalized linear model (GLM) estimates with L1 (LASSO) and/or L2 (ridge) penalties, or a combination are obtained using the library and function penalized (Goeman 2010).

To fit L2 penalized logistic regression models with a stepwise variable selection, we used the function plr in the package stepPlr (Park and Hastie 2008). We also used an $R$ implementation of SCAD, available at http://www.stat.umn.edu/ hzou/ ftpdir/code/one-step-SCAD-funs.R (accessed 05/09).

For linear and binary outcome data regression coefficients for penalized partial least squares were obtained using the function penali zed.pls in the library ppls (Krämer et al. 2008).

We used fivefold cross validation to select tuning parameters for all the methods that allowed that option.

Table 1 summarizes the algorithms and software packages.

\subsection{Simulated data}

\subsubsection{Continuous outcome data}

Each observation in a data set of size $n$ contains the predictors $\mathbf{X}=\left(X_{1}, \ldots, X_{p}\right)^{\prime}$, and the continuous outcome variable, $Y$. We assumed that only a small number of 
Table 1 Algorithms and software used in the simulation study

\begin{tabular}{ll}
\hline Algorithm & Software \\
\hline LASSO & R library lars \\
relaxed LASSO & R library relaxo \\
LARS & R library lars \\
elastic net & R library elasticnet \\
SCAD & http://www.stat.umn.edu/ hzou/ftpdir/code/ \\
& one-step-SCAD-funs.R (accessed 05/09) \\
GLM with L1 and/or L2 penalties & R library penalized \\
Penalized partial least squares & R library ppls \\
Logistic regr. w L2 penalty and stepwise selection & R library stepPlr \\
\hline
\end{tabular}

predictors $p^{*}<p$ are associated with $Y$ and denote those by $\mathbf{X}^{*}=\left(X_{1}^{*}, \ldots, X_{p^{*}}^{*}\right)^{\prime}$, a $1 \times p^{*}$ subvector of $\mathbf{X}$. For ease of exposition we let the predictors in $\mathbf{X}$ be ordered so that the first $p^{*}$ values of $\mathbf{X}$ correspond to $\mathbf{X}^{*}$. Given $\mathbf{X}^{*}$ and $\boldsymbol{\beta}^{*}=\left(\beta_{1}^{*}, \ldots, \beta_{p^{*}}^{*}\right)^{\prime}$, a $1 \times p^{*}$ vector with $\beta_{i}^{*} \neq 0$, the response $Y$ was generated from the linear model

$$
Y=\beta_{0}^{*}+\mathbf{X}^{* \prime} \boldsymbol{\beta}^{*}+\epsilon, \epsilon \sim \mathcal{N}\left(0, \sigma_{*}^{2}\right) .
$$

For each simulation, we then fit a linear model using all available predictors, $\mathbf{X}$, i.e. assuming

$$
Y=\beta_{0}+\mathbf{X}^{\prime} \boldsymbol{\beta}+\epsilon, \quad \epsilon \sim \mathcal{N}\left(0, \sigma^{2}\right),
$$

using the methods given in Sect. 2.1 and obtained the $1 \times p$ vector of parameter estimates $\hat{\boldsymbol{\beta}}$ of $\boldsymbol{\beta}$. In all settings we studied, $p=50$ and $p^{*}=10$ and the variance of the error term in Eq. (1) was $\sigma_{*}^{2}=1$. We also assessed the robustness of the methods by generating $\epsilon$ from a t-distribution with two degrees of freedom.

We generated $\mathbf{X}$ from a multivariate normal distribution, with mean $\mathbf{0}$ and correlation matrix $\Sigma_{X}$. To assess the impact of various correlation structures among the predictors on the performance of the methods, we studied several choices of $\Sigma_{X}=\left(\sigma_{i j}\right), i, j=$ $1, \ldots, p$. They include the independence correlation structure, $\Sigma_{X}=\mathbf{I}$, where I denotes the $p \times p$ identity matrix, a block diagonal structure for $\Sigma_{X}$, where each block submatrix has dimension $5 \times 5$ and constant entries $\sigma_{i j}=0.5, i \neq j$ for $|i-j| \leq 5$, and $\sigma_{i j}=0$ otherwise, and an autoregressive (AR) correlation structure for $\Sigma_{X}$ with $\sigma_{i j}=0.5^{|i-j|}$ for $|i-j| \leq 10$ and $\sigma_{i j}=0$ otherwise.

\subsubsection{Binary outcome data}

Binary data, labeled "controls" $(Y=0)$ and "cases" $(Y=1)$, were simulated similarly to the continuous outcomes. The probability $P(Y=1)$ was a function of the predictors $\mathbf{X}^{*}$, the $p^{*}$ dimensional subvector of $\mathbf{X}$ :

$$
\operatorname{logit}\left\{P\left(Y=1 \mid \mathbf{X}^{*}\right)\right\}=\beta_{0}^{*}+\mathbf{X}^{*^{\prime}} \boldsymbol{\beta}^{*},
$$


where $\operatorname{logit}(x)=\exp (x) /\{1+\exp (x)\}$. For each simulation we created a population of subjects by drawing $Y$ from a Bernoulli distribution with success probability given by model (3) with $\beta_{0}^{*}=-1$, given $\mathbf{X}$, and then sampled a fixed number of cases and controls to obtain a case-control study, a design popular in biological applications. Again, $p=50$ and $p^{*}=10$.

For each simulation, we obtained estimates $\hat{\boldsymbol{\beta}}$ by fitting a logistic model using all available predictors, $\mathbf{X}$, i.e. assuming

$$
\operatorname{logit}\{P(Y=1 \mid \mathbf{X})\}=\beta_{0}+\mathbf{X}^{\prime} \boldsymbol{\beta}
$$

using the methods given in Sect. 2.1.

We study multivariate normally distributed predictors $\mathbf{X}$ that have mean zero and $\Sigma_{X}=\mathbf{I}$, and also binary predictors $\mathbf{X}$, that is $X_{i}=0$ or $X_{i}=1$, with $P\left(X_{i}=1\right)=$ 0.5 .

\subsubsection{Parameter choices and sample sizes}

For simplicity, we assume that all outcome associated $\boldsymbol{\beta}^{*}=\left(\beta_{1}^{*}, \ldots, \beta_{p *}^{*}\right)$ coefficients in models (1) and (3) have the same magnitude, but half of them are positively and half of them are negatively associated with $Y$, i.e. the $\beta_{i}^{*}$ s differ by their sign. We chose $\beta_{i}^{*}=0.25,0.5$ and 1.0 for both linear and logistic models. For continuous outcomes the sample sizes were $n=100, n=200$, and $n=500$. For the binary outcome setting we used a case-control design with equal numbers of controls $\left(n_{0}\right)$ and cases $\left(n_{1}\right)$ with $n_{0}=n_{1}=100, n_{0}=n_{1}=200$, and $n_{0}=n_{1}=500$. All simulations and analyses were implemented in $\mathrm{R}$.

\subsection{Analysis}

We assessed the performance of two strategies to obtain parameter estimates and their standard errors for both linear and logistic regression models.

\subsubsection{Linear regression}

\section{Algorithm 1 (Adaptive approach)}

This is a one-stage approach that uses the estimates $\hat{\boldsymbol{\beta}}$ obtained from the respective procedure. We denote the vector of coefficients of $\hat{\boldsymbol{\beta}}$ that are either the intercept or are non-zero by $\hat{\boldsymbol{\beta}}_{\text {adapt }}$, and by $\hat{X}_{S i}$ the vector of predictors for the $i^{t h}$ subject corresponding to the intercept and the non-zero parameter estimates. The corresponding $p_{\text {adapt }} \times n$ design matrix is $\hat{\mathbf{X}}_{S}$. We let $\hat{\sigma}_{\text {adapt }}^{2}$ be the mean squared error of the fit for $\hat{\boldsymbol{\beta}}$, but with the degrees of freedom $n-p_{\text {adapt }}$, where $p_{\text {adapt }}$ is the dimension of $\hat{\boldsymbol{\beta}}_{\text {adapt }}$. The covariance matrix of $\hat{\boldsymbol{\beta}}_{\text {adapt }}$ is estimated as

$$
\widehat{\operatorname{cov}}\left(\hat{\boldsymbol{\beta}}_{\text {adapt }}\right)=\hat{\sigma}_{\text {adapt }}^{2}\left(\hat{\mathbf{X}}_{S} \hat{\mathbf{X}}_{S}^{\prime}\right)^{-1}
$$




\section{Algorithm 2 (Oracle approach)}

This is a two stage approach. First we obtain $\hat{\mathbf{X}}_{S}$ as in Algorithm 1. In stage two we regress $Y$ on $\hat{\mathbf{X}}_{S}$ to get a $p_{\text {adapt }} \times 1$ vector $\hat{\boldsymbol{\beta}}_{\text {oracle }}$ of new parameter estimates, which include an intercept. We let $\hat{\sigma}_{\text {oracle }}^{2}$ be the mean squared error of the fit when $\hat{\boldsymbol{\beta}}_{\text {oracle }}$ is used, with $n-p_{\text {adapt }}$ degrees of freedom. The estimated covariance matrix of $\hat{\boldsymbol{\beta}}_{\text {oracle }}$ is then

$$
\widehat{\operatorname{cov}}\left(\hat{\boldsymbol{\beta}}_{\text {oracle }}\right)=\hat{\sigma}_{\text {oracle }}^{2}\left(\hat{\mathbf{X}}_{S} \hat{\mathbf{X}}_{S}^{\prime}\right)^{-1} .
$$

\subsubsection{Logistic regression}

Like for linear regression, $\hat{X}_{S i}$ is the vector of predictors for the $i^{\text {th }}$ subject corresponding to the intercept and the non-zero components of $\hat{\boldsymbol{\beta}}$ and $\hat{\mathbf{X}}_{S}$ is the corresponding design matrix.

Algorithm 1 (Adaptive approach)

Again, $\hat{\boldsymbol{\beta}}_{\text {adapt }}$ denotes the vector of coefficients of $\hat{\boldsymbol{\beta}}$ obtained from the respective procedure that are either the intercept or are non-zero. Letting logit $\left(\hat{p}_{i}^{a}\right)=\hat{X}_{\text {Si }}^{\prime} \hat{\boldsymbol{\beta}}_{\text {adapt }}$, $\hat{\mathbf{p}}_{\text {adapt }}=\left(\hat{p}_{1}^{a}\left(1-\hat{p}_{1}^{a}\right), \ldots, \hat{p}_{n}^{a}\left(1-\hat{p}_{n}^{a}\right)\right)$ and $\hat{V}_{\text {adapt }}=\hat{\mathbf{p}}_{\text {adapt }} \mathbf{I}$, where $\mathbf{I}$ denotes the identity matrix, we compute the covariance matrix of the estimates as

$$
\widehat{\operatorname{cov}}\left(\hat{\boldsymbol{\beta}}_{\text {adapt }}\right)=\left(\hat{\mathbf{X}}_{S} \hat{V}_{\text {adapt }} \hat{\mathbf{X}}_{S}^{\prime}\right)^{-1}
$$

\section{Algorithm 2 (Oracle approach)}

First we obtain $\hat{\mathbf{X}}_{S}$, and then compute $\hat{\boldsymbol{\beta}}_{\text {oracle }}$ by re-fitting the standard logistic regression model with $\hat{\mathbf{X}}_{S}$ instead of $\mathbf{X}$ to the outcome data. Letting logit $\left(p_{i}^{o}\right)=$ $\hat{X}_{\text {Si }}^{\prime} \hat{\boldsymbol{\beta}}_{\text {oracle }}, \hat{\mathbf{p}}_{\text {oracle }}=\left(\hat{p}_{1}^{o}\left(1-\hat{p}_{1}^{o}\right), \ldots, \hat{p}_{n}^{o}\left(1-\hat{p}_{n}^{o}\right)\right)$ and $\hat{V}_{\text {oracle }}=\hat{\mathbf{p}}_{\text {oracle }} \mathbf{I}$, where $\mathbf{I}$ denotes the identity matrix, we compute the covariance matrix of the estimates as

$$
\widehat{\operatorname{cov}}\left(\hat{\boldsymbol{\beta}}_{\text {oracle }}\right)=\left(\hat{\mathbf{X}}_{S} \hat{V}_{\text {oracle }} \hat{\mathbf{X}}_{S}^{\prime}\right)^{-1}
$$

\subsection{Performance criteria}

We evaluated the influence of sample size, $n$, and magnitude of the associations $\boldsymbol{\beta}^{*}$ on each method's ability to identify the true outcome associated predictors $\mathbf{X}^{*}$ and on the two algorithms described above to estimate the corresponding regression parameters $\beta^{*}$.

\subsubsection{Performance criteria for variable selection}

False positives $(F P s)$ Let $\boldsymbol{\beta}=\left(\beta_{0}, \beta_{1}, \ldots, \beta_{p}\right)^{\prime}=\left(\boldsymbol{\beta}^{*}, \mathbf{0}\right)^{\prime}$, where $\mathbf{0}$ is a $1 \times\left(p-p^{*}\right)$ vector of zeros. A FP occurs for $\beta_{j}$ when $\beta_{j}=0$ but its regularized estimate $\hat{\beta}_{j} \neq 0$. The FP rate for $\beta_{j}$ is the percentage of times an FP occurs for $\beta_{j}$, and the overall FP rate is the average of the FP rates across all zero coefficients of $\boldsymbol{\beta}$. 
False Negatives (FNs) A FN occurs for $\beta_{j}$ when $\beta_{j} \neq 0$ but its regularized estimate $\hat{\beta}_{j}=0$. The FN rate for $\beta_{j}$ is the percentage of times a FN occurs for $\beta_{j}$, and the overall $\mathrm{FN}$ rate is the average of the $\mathrm{FN}$ rates across all non-zero coefficients of $\boldsymbol{\beta}$.

\subsubsection{Impact of model selection on parameter estimates, coverage computations}

The following coverages of the $95 \%$ confidence intervals (CIs) for linear and logistic models were computed. The coverage of zeros is the number of times that either the regularized estimate of a $\beta_{j}=0$ coefficient is zero, i.e. $\hat{\beta}_{j}=0$, or the $95 \% \mathrm{CI}$ of $\hat{\beta}_{j} \neq 0$ includes zero divided by the number of $p-p^{*}$ of zero coefficients. The $95 \%$ CIs are computed using the asymptotic approximation, the normal distribution, and the standard errors from either Algorithm 1 or Algorithm 2. We compute the coverage of zeros separately for the zero and non-zero coefficients of $\boldsymbol{\beta}$ and report the average over all $\beta_{j}=0$ and $\beta_{j}=\beta_{j}^{*} \neq 0$ respectively.

The coverage of the true $\beta^{*}$ coefficients is the number of times that the $95 \% \mathrm{CI}$ around $\hat{\beta}_{\text {adapt }}$ or $\hat{\beta}_{\text {oracle }}$ includes the true value of $\beta_{j}^{*}\left(=\beta_{j} \neq 0\right)$ divided by the number $p^{*}$ of non-zero coefficients $\boldsymbol{\beta}^{*}$. Again, we report the average over all coefficients $\beta_{j}^{*}, j=1, \ldots, p^{*}$.

\section{Results}

\subsection{Results for linear regression}

\section{LARS}

The $F P$ rate ranged from 11.1 to $34.8 \%$, and was slightly lower for the block correlation structure than the AR or independent correlations (Fig. 1 and Supplemental Table 1). The $F N$ rate was below 0.05 for sample sizes $n=200$ and $n=500$ and around $20 \%$ for $n=100$ for all effect sizes and correlations. The coverage of zero for $\beta_{j}=0$ was close to $100 \%$ for Algorithm 1 and around $95 \%$ for Algorithm 2. The coverage of zero for $\beta^{*} \neq 0$ was $0 \%$ for both algorithms for $n=500$ for all effect sizes (Fig. 2). The $95 \%$ CI coverage of $\beta^{*}$ for the $\hat{\beta} \neq 0$ coefficients was around $95 \%$ for Algorithm 2 with $n=500$. It also was around $95 \%$ for both algorithms for $n=200$ and $n=500$ for the block correlation structure, but for all other correlations Algorithm 1 had lower coverage than Algorithm 2, generally below $90 \%$ (Fig. 3).

\section{LASSO}

Similar to $L A R S$, the $F P$ rate was slightly lower for the block correlation structure than the AR or independent correlations (Fig. 1 and Supplemental Table 2), and it ranged from 10.3 to $44.6 \%$. The $F N$ rate was below $5 \%$ for $n=200$ and $n=500$, and for $n=100$ with $\beta^{*}=0.5$ and $\beta^{*}=1$. The coverage of zero for $\beta_{j}=0$ was close to $100 \%$ for Algorithm 1 and around $95 \%$ for Algorithm 2 for all sample and effect sizes. The coverage of zero for $\beta^{*}$ was $0 \%$ for both algorithms for $n=500$ for all effect sizes (Fig. 2). The coverage of $\boldsymbol{\beta}^{*}$ for $\hat{\beta} \neq 0$ estimates was slightly lower for Algorithm 1 than 2. Algorithm 2 had $95 \%$ coverage for $n=500$ and slightly below $95 \%$ for $n=200$ (Fig. 3). Algorithm 1 had somewhat higher, 

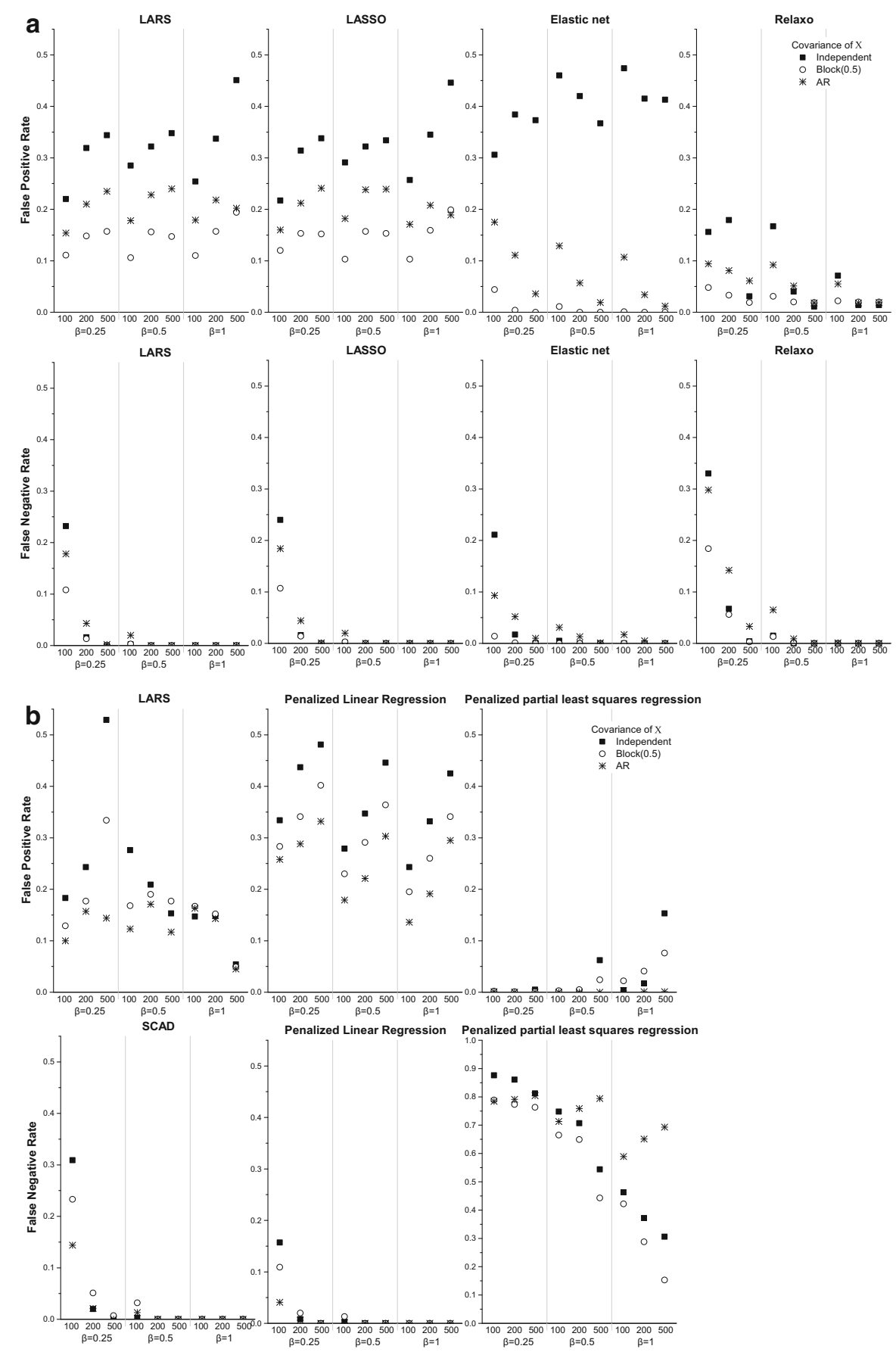

Fig. 1 False positive $(F P)$ and false negative $(F N)$ rates for LARS, LASSO, elastic net and relaxo (a), and SCAD, penalized linear regression and penalized partial least squares regression (b) 


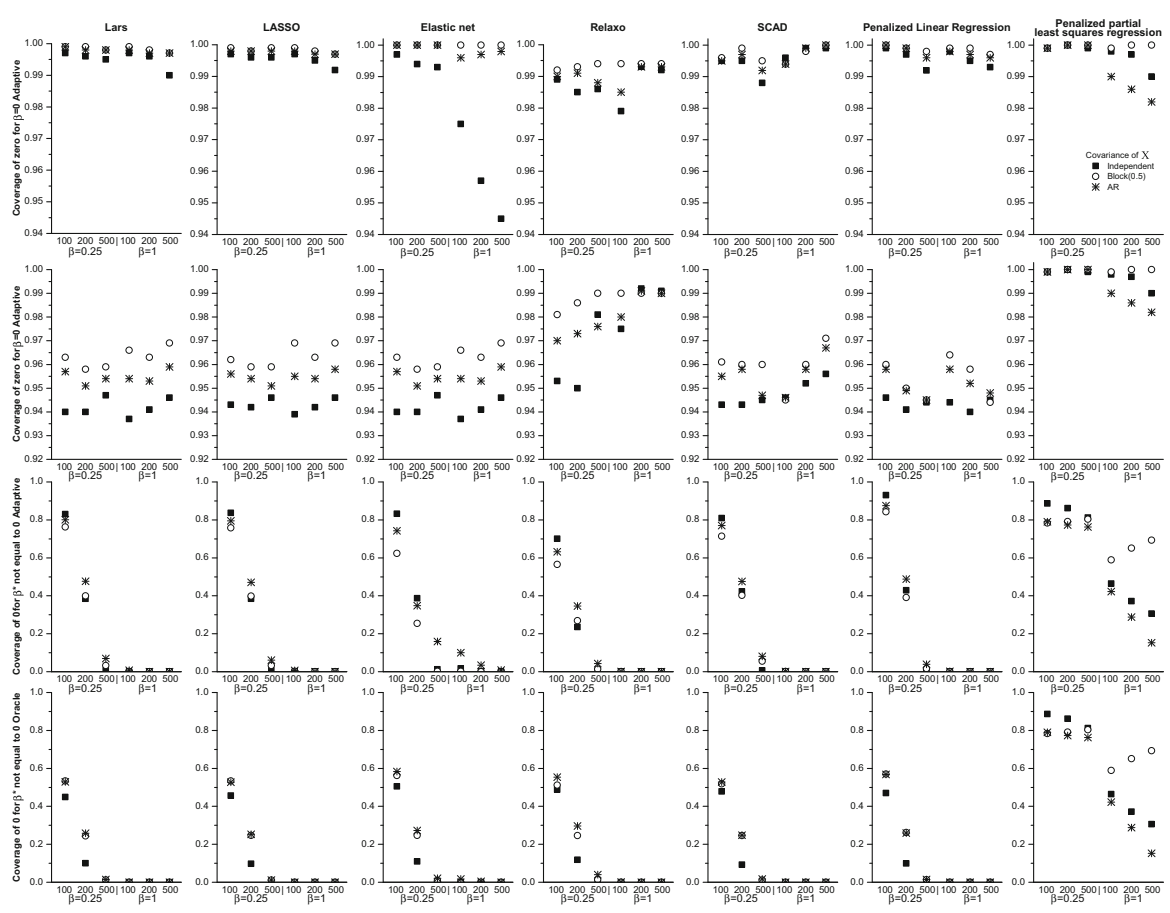

Fig. 2 Coverage of zero for $\beta=0$ for the adaptive and oracle confidence intervals (top two rows) and coverage of zero for $\beta^{*} \neq 0$ (bottom two rows)

albeit still less than $95 \%$ coverage for the block correlation structure than the other correlations.

\section{Elastic net}

The FP rate ranged from 30.6 to $47.4 \%$ for independent predictors, while it was below $4 \%$ for the block correlation structure for all values of $n$ and $\beta^{*}$ (Table 4). For the AR correlation structure the FP rate was less than $5 \%$ for $n=500$ for all values of $\beta^{*}$. The $F N$ rate was low for all correlation structures, and less than $5 \%$ for $n=200$ and 500, regardless of the effect sizes (Fig. 1 and Supplemental Table 3). The coverage of the zero coefficients was close to $100 \%$ for both algorithms. The coverage of zero for coefficients corresponding to $\beta^{*}$ was $0 \%$ for both algorithms for $n=500$ for all effect sizes (Fig. 2). Overall, the coverage of $\beta^{*}$ for $\hat{\beta} \neq 0$ coefficients was noticeably higher for Algorithm 2 than for Algorithm 1. Algorithm 2 had close to $95 \%$ coverage with the exception of small sample sizes. For $n=100$ with $\beta^{*}=0.25$ the coverage fell below $90 \%$, likely due to variables not being selected (Fig. 3).

\section{Relaxo}

The FP and FN rates were slightly lower for the block correlation structure than the independent or AR correlations, but were less than $5 \%$ for $n=200$ and $n=500$ for all effect sizes and correlations (Fig. 1 and Supplemental Table 4). Both FP and FN 

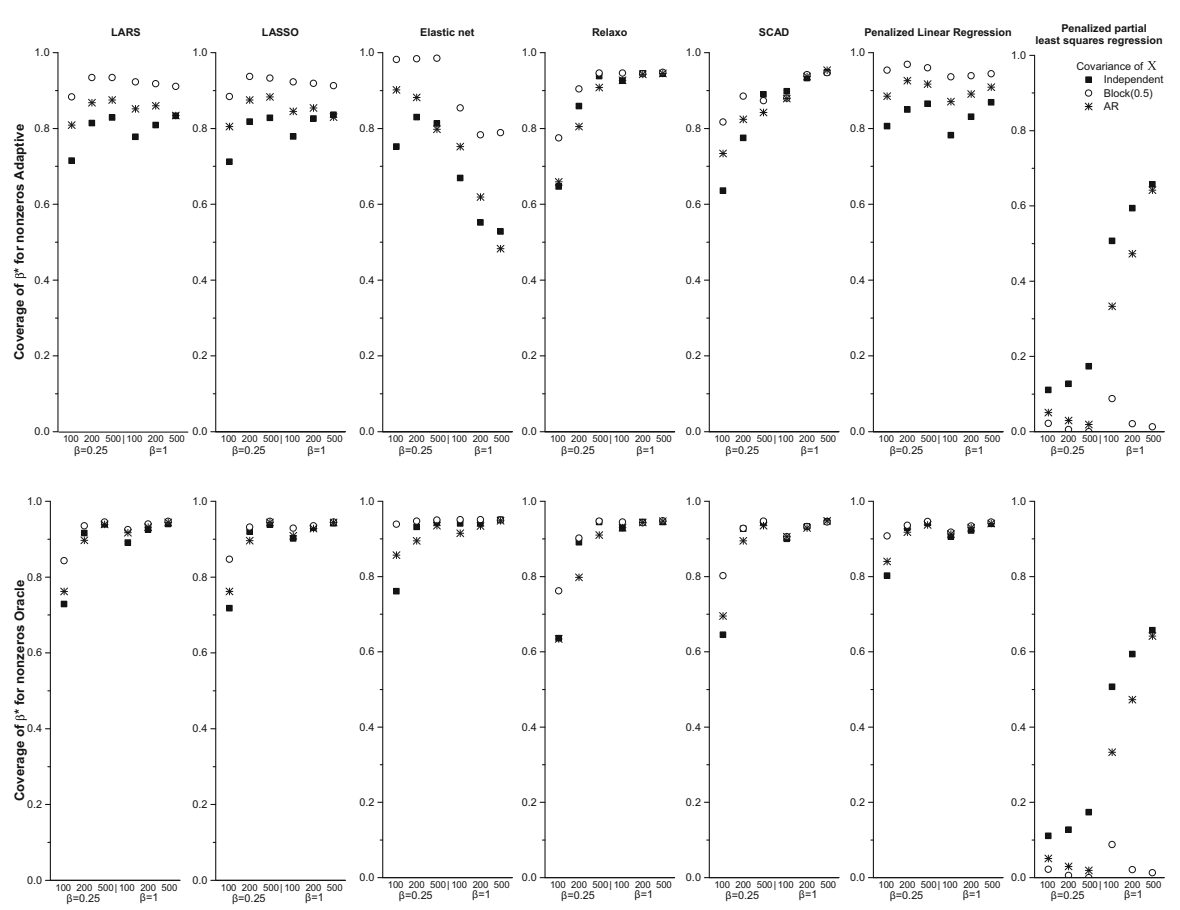

Fig. 3 Coverage of the $95 \%$ CIs computed based on the adaptive (Algorithm 1) or oracle (Algorithm 2) variance estimates of $\beta^{*}$ for $\hat{\beta} \neq 0$

rates also dropped quickly as $n$ increased. For example, for the independent correlation structure and $\beta^{*}=0.25$ the $F P$ and $F N$ rates were 15.6 and $33.0 \%$ for $n=100$ and 3.1 and $0.4 \%$ for $n=500$, respectively. For all correlation structures the coverage of zero for $\beta=0$ was close to $100 \%$ for both algorithms. The coverage of zero for $\beta^{*}$ was close to $0 \%$ for both algorithms for $n=500$ for all effect sizes (Fig. 2). The coverage of $\beta^{*}$ for $\hat{\beta} \neq 0$ coefficients was similar for Algorithms 1 and 2, and close to $95 \%$ for $n=500$ for all effect sizes, and for $n=200$ with $\beta^{*}=0.5$ and $\beta^{*}=1.0$ for all correlation structures (Fig. 3).

\section{$S C A D$}

The FP rates were very similar for all correlation structures and less than $5 \%$ for $n=500$ with $\beta^{*}=1$. The $F N$ rate was generally low and dropped quickly as $n$ increased (Fig. 1 and Supplemental Table 5). For $n=200$ or $n=500$ it was less than $5 \%$ for all values of $\beta^{*}$. It was greater than $10 \%$ only for $n=100$ with $\beta^{*}=0.25$. The coverage of zero for $\beta=0$ was close to $100 \%$ for Algorithm 1 and $95 \%$ for Algorithm 2. The coverage of zero for $\beta^{*}$ was $0 \%$ for both algorithms for $n=500$ for all effect sizes (Fig. 2). For all correlation structures the coverage of the $95 \%$ CIs of $\beta^{*}$ for $\hat{\beta} \neq 0$ coefficients was close to $95 \%$ for $n=500$ for Algorithm 2 for all effect sizes, and for Algorithm 1 for $n=500$ with $\beta^{*}=1$. Again, the coverage was noticeably higher for Algorithm 2 than for Algorithm 1 and it increased for both algorithms with sample and effect size (Fig. 3). 


\section{Penalized penalized linear regression}

The FP rate ranged from $17.9 \%$ for $n=100$ with $\beta^{*}=0.5$ and the block correlation structure to $48.5 \%$ for $n=100$ with $\beta^{*}=0.25$ and independent predictors (Fig. 1 and Supplemental Table 6). The $F N$ rate was $16.2 \%$ for $n=100, \beta^{*}=0.25$ for the independent correlation structure, but for all other $n$ and effect sizes it was less than $1 \%$. For all correlation structures the coverage of zero for the $\beta=0$ coefficients for Algorithm 1 was higher than $99 \%$ for all sample sizes and effect sizes, while for Algorithm 2 the coverage was around $95 \%$. The coverage of zero for $\beta^{*}$ was $0 \%$ for both algorithms for $n=500$ for all values of $\beta^{*}$. For independent $\mathbf{X}$, the coverage of $\beta^{*}$ of the $95 \%$ CIs for $\hat{\beta} \neq 0$ coefficients ranged from 78.7 to $86.6 \%$ for Algorithm 1. It was around $95 \%$ for Algorithm 2 only for $n=500$, but lower for $n=100$ and $n=200$. Similar patterns were seen for the AR correlation structure. For the block correlation structure both algorithms had close to $96 \%$ coverage for $n=200$ and $n=500$ for all effect sizes (Fig. 3).

\section{Partial least squares}

The $F P$ rate was less than $5 \%$ for most correlation structures and effect sizes, the only outlier was the $F P$ value of $15.3 \%$ for $n=500$ and $\beta^{*}=1$ with the independent correlation structure (Fig. 1 and Supplemental Table 7). However, the $F N$ rate ranged from $15.3 \%$ for $n=500, \beta^{*}=1$ and the AR correlation structure to $87.6 \%$ for $n=$ 100 with $\beta^{*}=0.25$ for independent predictors, and was above $60 \%$ for all correlation structures and most sample sizes. The $F N$ rate was lower for larger effect and sample sizes. The coverage of zero for $\beta_{j}=0$ was around $100 \%$ for both algorithms for settings. The coverage of zero for $\beta^{*}$ for $n=500$ ranged from 15.3 to $81.2 \%$, and was not different for the two algorithms (Fig. 2). For both algorithms the coverage of $\beta^{*}$ of the $95 \%$ CIs for $\hat{\beta} \neq 0$ was very low for all correlation structures, ranging from 0 to $65.7 \%$ (for independent $\mathbf{X}$, with $n=500$ and $\beta^{*}=1$ ) (Fig. 3).

\subsubsection{Non-normal error distribution}

When we generated outcome data from a linear model (1) where the error term $\epsilon$ followed a t-distribution with 2 degrees of freedom for independent $\mathbf{X}$ (Table 2), the FP rate was lower for LARS, LASSO, elastic net and relaxo while for these methods the $F N$ rate was higher compared to normally distributed errors. In contrast, for SCAD, the FP rate was much higher and the $F N$ rate much lower than for normal errors. Simulation runs based on penalized partial least squares regression failed to give reasonable results in so many instances that we do not present any results for this method.

For all methods the coverage of zero for the $\beta=0$ coefficients for Algorithms 1 and 2 was very similar to the normal case. For all methods the coverage of zero for $\beta^{*}$ was much higher than for normally distributed errors. The coverage of $\beta^{*}$ of the $95 \%$ CIs for $\hat{\beta} \neq 0$ coefficients however was much lower than in the normal case for Algorithm 1 and Algorithm 2 and much below the nominal $95 \%$. When the errors were generated from a t-distribution with 15 degrees of freedom however (Supplemental Table 8), the coverage was much improved and similar to that seen for normally distributed error terms. 


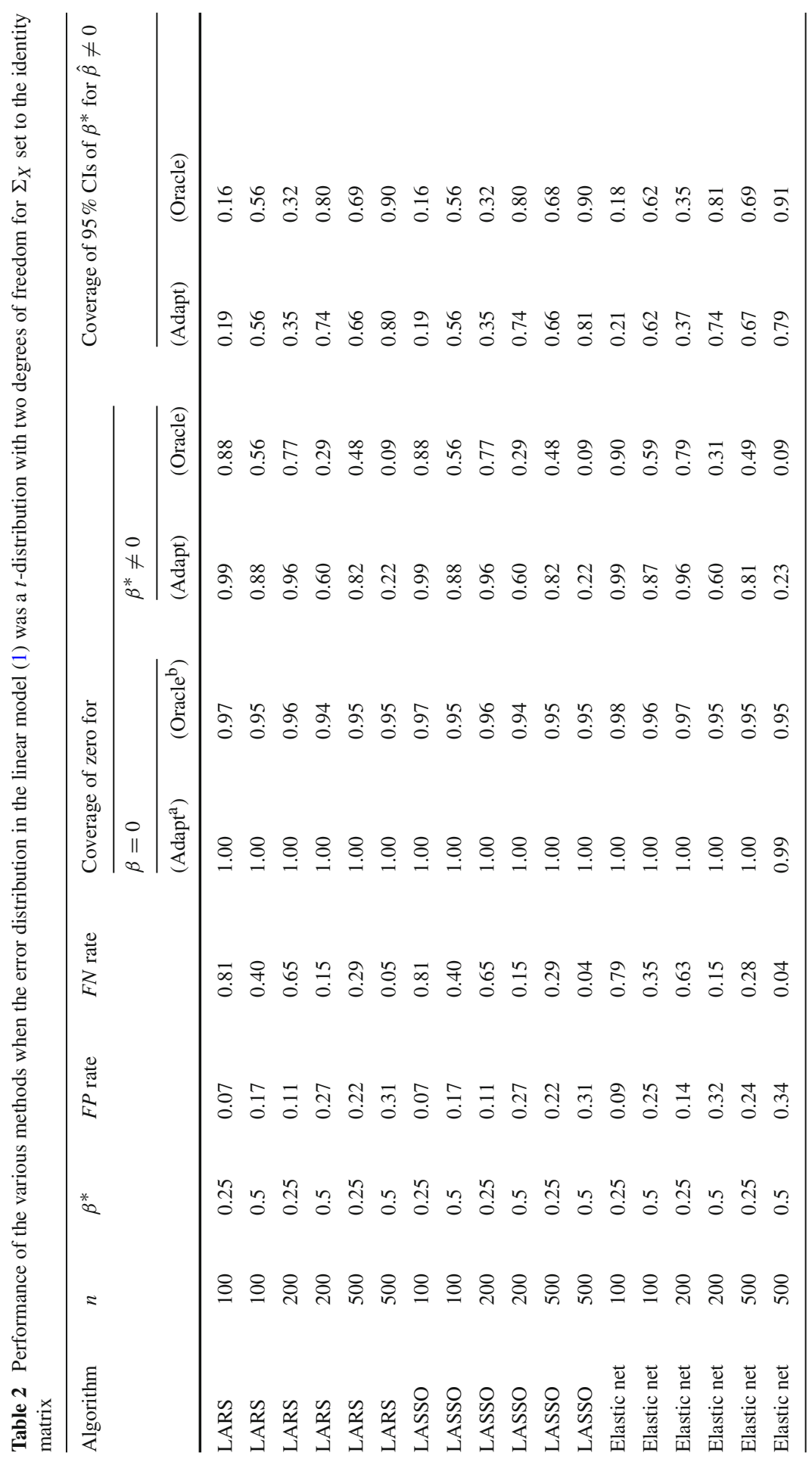




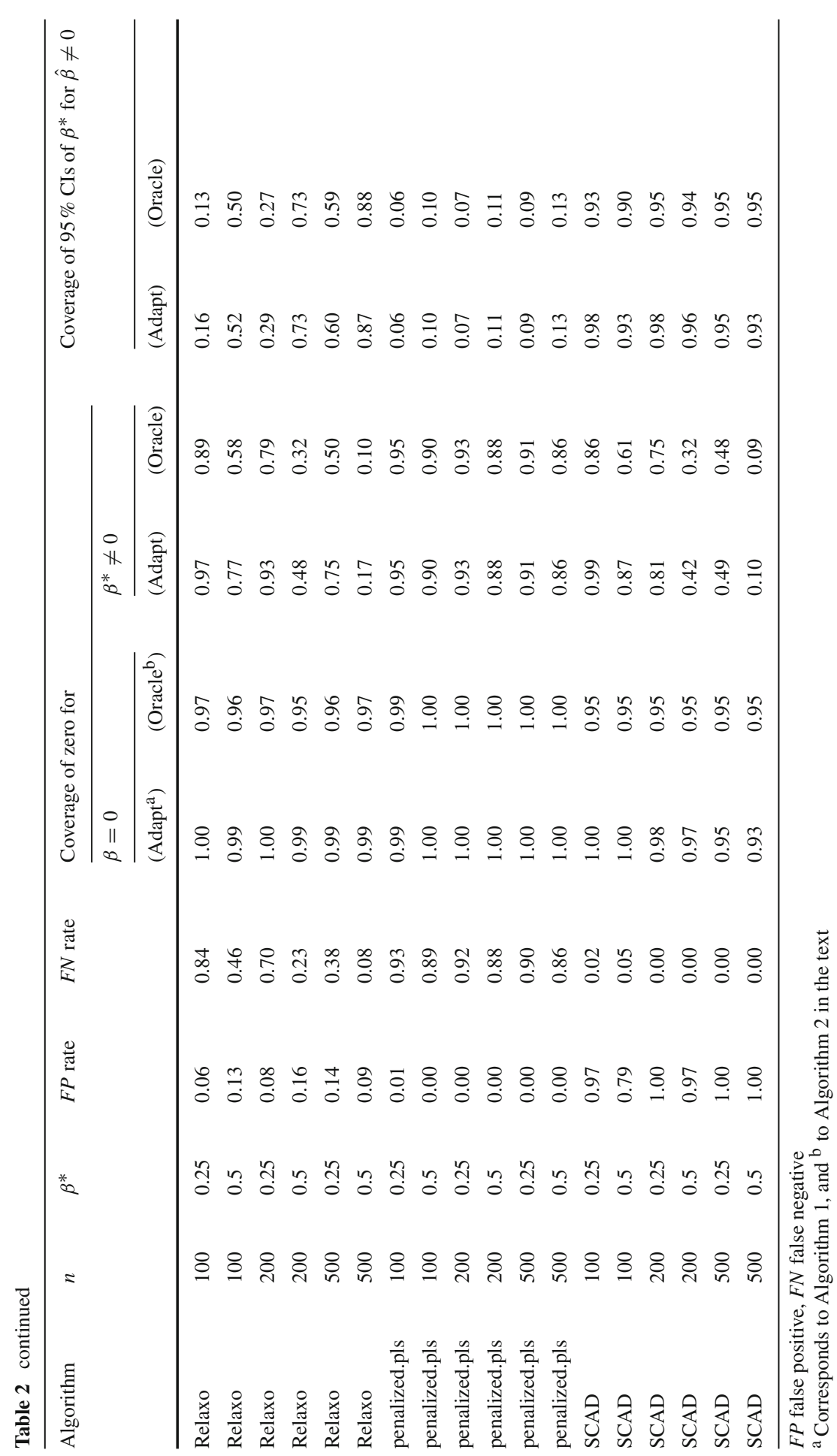




\subsubsection{Results for $p>n$}

We also attempted to assess the performance of the methods when $p>n$ by generating data with $p=500$ and $p^{*}=10$ for $n=100,200$ and $n=500$ for independent predictors $\mathbf{X}$. SCAD, elastic net and penalized linear regression resulted in so many error messages that we do not present any findings for these algorithms.

Results for LARS, LASSO and relaxo are given in Table 3. For both LARS and LASSO, the $F P$ rate was lower than for the $p<n$ setting for $n=100$ and 200, but was above $74 \%$ for $n=500$ for all values of $\beta^{*}$ The $F N$ rate was low except for $\beta^{*}=0.25$ with $n=100$. The coverage of zero for the $\beta=0$ coefficients for Algorithm 1 was higher than $99 \%$ for all sample sizes and effect sizes, while for Algorithm 2 the coverage was below $70 \%$. The coverage of zero for $\beta^{*}$ ranged from 0 to $93 \%$ for Algorithm 1 and from 0 to $26 \%$ for Algorithm 2. The coverage of $\beta^{*}$ of the $95 \%$ CIs for $\hat{\beta} \neq 0$ coefficients was less than $9 \%$ for both algorithms.

For relaxo, the $F P$ and $F N$ rates were similar to those seen for LARS and LASSO for $n=100$ and $n=200$, with the exception of the $F P$ rates for $n=500$, which were less than $2 \%$. The coverage of zero for the $\beta=0$ coefficients for Algorithm 1 ranged from 2 to $83 \%$, for all sample sizes and effect sizes, while for Algorithm 2 the coverage was below $62 \%$. The coverage of zero for $\beta^{*}$ was below $5 \%$ for $\beta^{*}=0.5$ and $\beta^{*}=1$ for both algorithms. The coverage of $\beta^{*}$ of the $95 \%$ CIs for $\hat{\beta} \neq 0$ coefficients was less than $10 \%$ for both Algorithm 1 and 2 .

\subsubsection{Summary of results for linear regression}

The estimation methods that had a high false positive (FP) rate were LARS, LASSO, elastic net, SCAD and penalized linear regression. Not surprisingly, the FN rate of these methods was low. Partial least squares regression had a low FP rate at the cost of having many false negatives. Only relaxo had both a low FP and FN rate. The coverage of zero for the $\beta=0$ coefficients for Algorithm 1 was close to $100 \%$ for all methods, while for Algorithm 2 it was closer to $95 \%$. The coverage of zero of the $\beta^{*}$ coefficients was close to zero for all methods with the exception of penalized least squares (Fig. 3). The coverage of the true $\beta^{*}$ coefficients of the $95 \%$ CIs around $\hat{\beta} \neq 0$ was typically higher for Algorithm 2 than for Algorithm 1. For Algorithm 2 it was close to $95 \%$ for large sample sizes and effect sizes for all methods with the exception of penalized partial least squares, for which coverage even for $n=500$ with $\beta^{*}=1$ was around $65 \%$. When $p>n$, the coverage of both algorithms was much lower than $95 \%$, however.

\subsection{Results for logistic regression}

\section{LASSO}

The $F P$ rate was somewhat higher for binary predictors than for independent normally distributed $\mathbf{X}$, but for both it was appreciable, with values up to $44 \%$ even for large effect and sample sizes (Table 4 ). The $F N$ rate was above $50 \%$ for $\beta^{*}=0.25$, but was less than $6 \%$ for binary predictors with $\beta^{*}=1.0$ for all sample sizes, for $\beta^{*}=0.5$ 


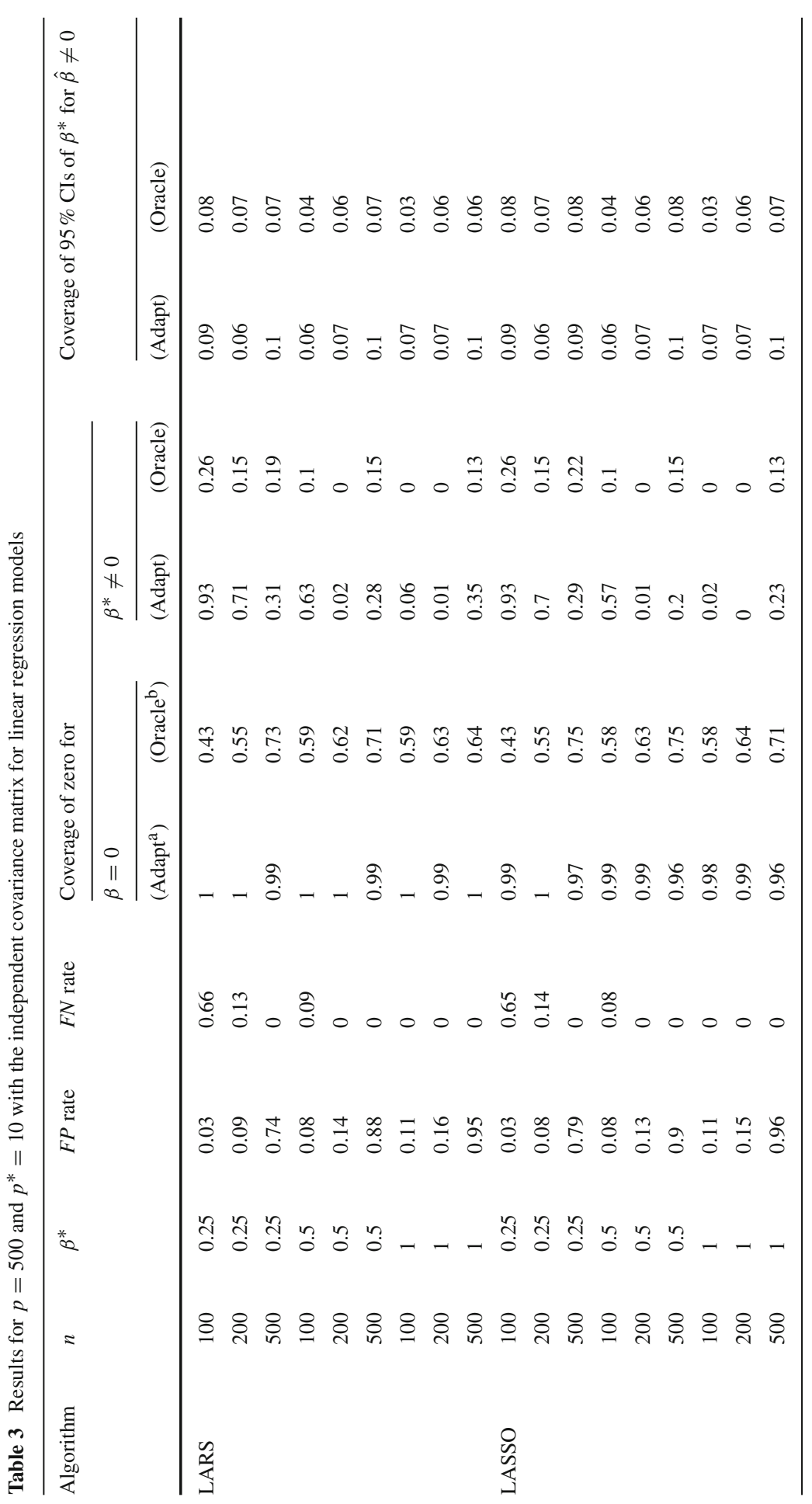




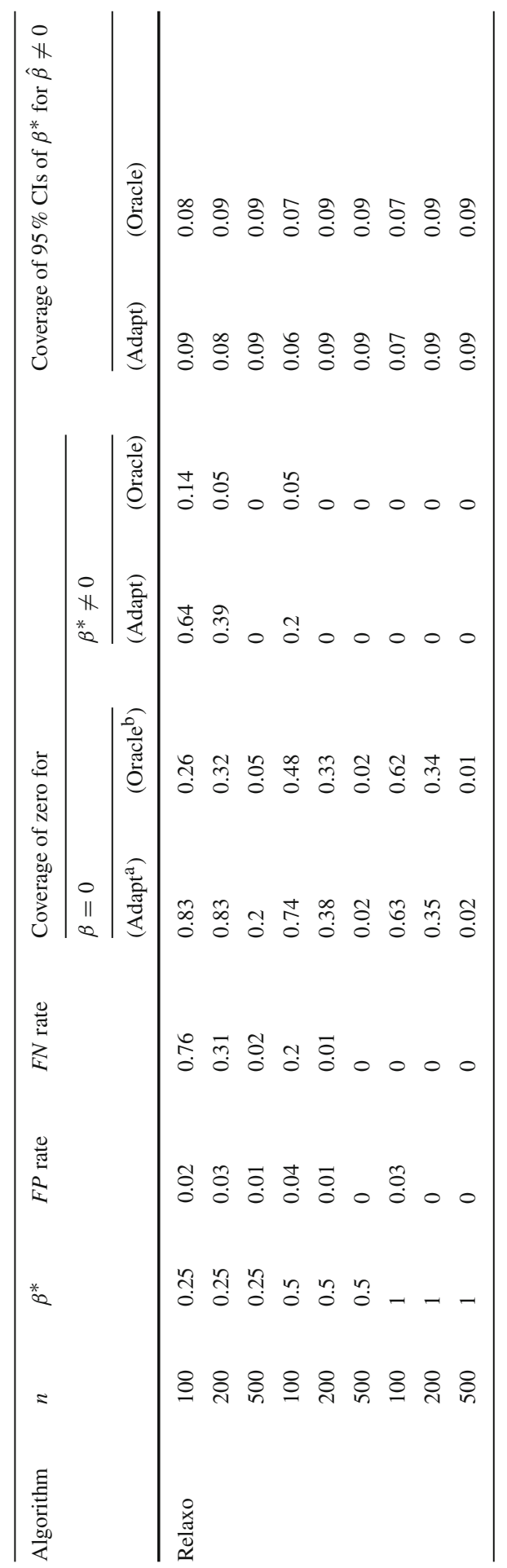




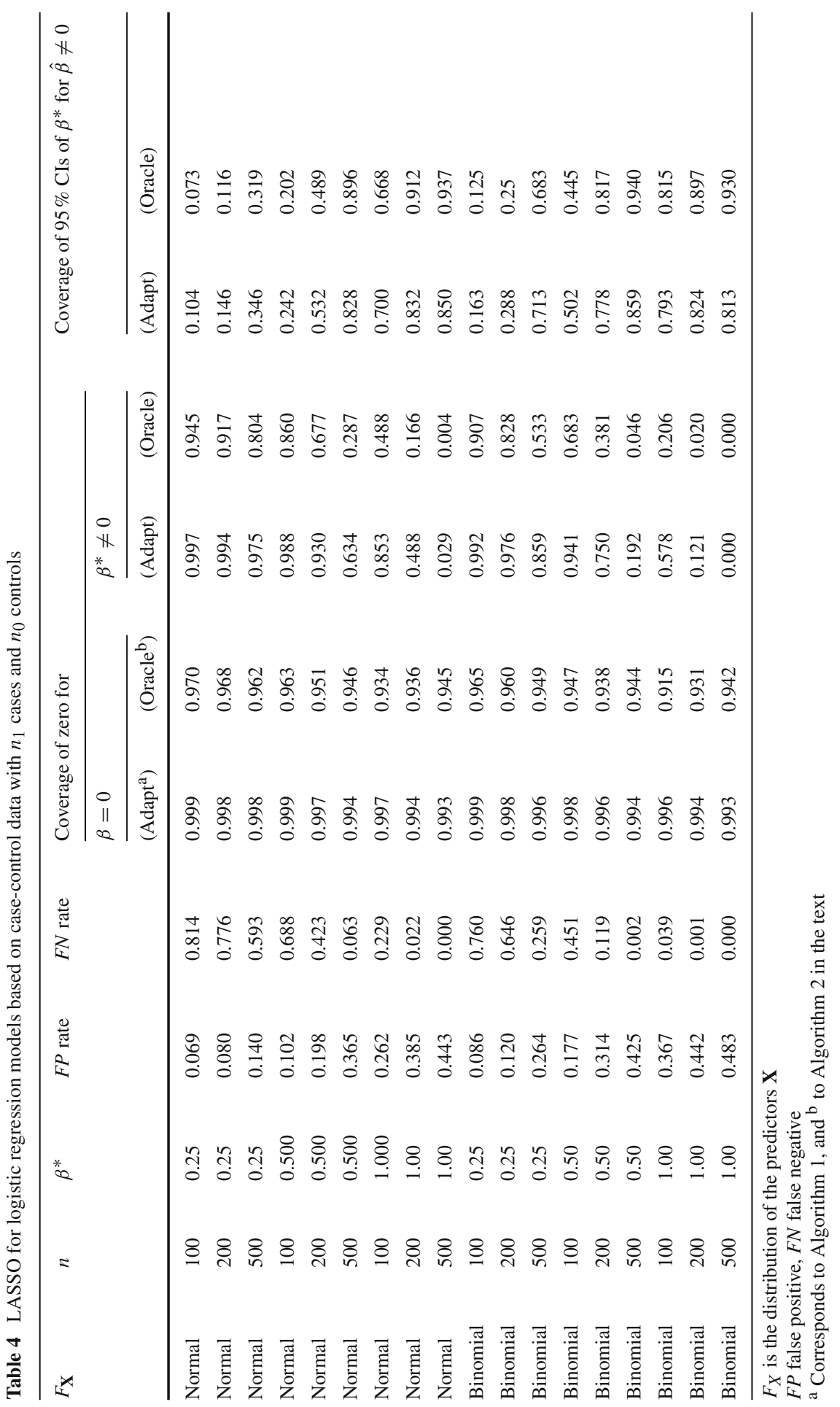


for $n=200$ and 500, and for normally distributed $\mathbf{X}$ with $\beta^{*}=1.0$ for $n=200$ and 500. The coverage of zero for $\beta=0$ was nearly $100 \%$ for Algorithm 1 and closer to $95 \%$ for Algorithm 2. The coverage of zero for $\beta^{*}$ was higher for Algorithm 1 than Algorithm 2. For Algorithm 1 the coverage of zero for $\beta^{*}$ ranged from $0 \%$ for $n=500$ with $\beta^{*}=1$ and binary predictors to $99.7 \%$ for $n=100$ with $\beta^{*}=0.25$. The coverage of $\beta^{*}$ of the $95 \%$ CIs around $\hat{\beta} \neq 0$ was very low for both algorithms, with the exception of $n=500$ and $\beta^{*}=1.0$ for normally distributed and $\beta^{*}=0.5$ and $\beta^{*}=1.0$ for binary $\mathbf{X}$, where coverage was close to $95 \%$.

\section{$S C A D$}

For both, independent normally distributed and binary $\mathbf{X}$ the FP rate was very low; the largest value was $5 \%$ for $n=500$ with $\beta^{*}=0.5$, while the $F N$ rate was extremely high, with values above $80 \%$ for many other settings (Table 5). Only for $n=500$ with $\beta^{*}=1.0$ and for binary predictors also for $\beta^{*}=0.5$ was the $F N$ rate below $15 \%$. The coverage of zero for $\hat{\beta}_{j}=0$ was nearly $100 \%$ for both algorithms. The coverage of zero for $\beta^{*} \neq 0$ was similar for both algorithms and ranged from 0.04 to $99.6 \%$. It dropped as sample size and effect size increased. The coverage of $\beta^{*}$ of the $95 \% \mathrm{CIs}$ around $\hat{\beta} \neq 0$ was very low for both algorithms, with the exception of $n=500$ and $\beta^{*}=1.0$ for both normally distributed and binary predictors, for which the coverage was approximately $93 \%$.

\section{Penalized logistic regression}

For the independent normally distributed and binary predictors $\mathbf{X}$ the $F P$ rate was similar, and ranged from 44.2 to $72.0 \%$. We observed an FP of $45.3 \%$ for independent normal $\mathbf{X}$ even for $\beta^{*}=1$ and $n=500$ (Table 6 ). The $F N$ rate depended more strongly on the effect size, was somewhat higher for normally distributed $\mathbf{X}$ but in all cases decreased noticeably as $n$ increased. For example, for normally distributed predictors with $\beta^{*}=0.5$, the $F N$ rate was $28.3 \%$ for $n=100,13.4 \%$ for $n=200$ and $3 \%$ for $n=500$. The coverage of zero for $\beta=0$ was nearly $100 \%$ for Algorithm 1 and between 91.5 and $94.8 \%$ for Algorithm 2. The coverage of zero for $\beta^{*}$ ranged from from 0.0 to $99.9 \%$ for Algorithm 1 and was slightly lower for Algorithm 2. It dropped as sample size and effect size increased for both algorithms. The coverage of $\beta^{*}$ of the $95 \%$ CIs around $\hat{\beta} \neq 0$ was slightly lower for Algorithm 1 than 2 . For $\beta^{*}=0.5$ and $\beta^{*}=1.0$ with $n=500$ Algorithm 2 had a coverage of nearly $95 \%$.

Adaptive logistic regression

For all $\mathbf{X}$ the $F P$ rate was less than $10 \%$ for $n=200$ and $n=100$ for all effect sizes, while the $F N$ rates for those $n$ ranged from 67 to $85 \%$ (Table 7). For $n=500$, the $F P$ rate was $5 \%$ for $\beta^{*}=0.25$ and 20 and $22 \%$ for $\beta^{*}=0.5$ and $\beta^{*}=1$ respectively, with corresponding $F N$ rates of 77, 22 and $0 . \%$. For binary predictors the $F P$ rate was higher, and ranged from 6 to $23 \%$, and $F N$ rates ranged from 0 to $81 \%$. The coverage of zero for $\beta=0$ was nearly $100 \%$ for Algorithm 1 for sample sizes $n=100$ and $n=200$. For $n=500$ with effect sizes $\beta^{*}=0.5$ and $\beta^{*}=1.0$ the coverage for Algorithm 1 was $95 \%$. The coverage of zero for $\beta^{*}$ ranged from from 0.0 to $99 \%$ for Algorithm 1 and was slightly lower for Algorithm 2. It dropped as sample size and effect size increased for both algorithms. The coverage of $\beta^{*}$ of the $95 \%$ CIs around $\hat{\beta} \neq 0$ was slightly lower for Algorithm 1 than 2. However, Algorithm 2 had $94 \%$ coverage only for $n=500$ and $\beta^{*}=1.0$ and $\beta^{*}=0.5$. For all other sample and 


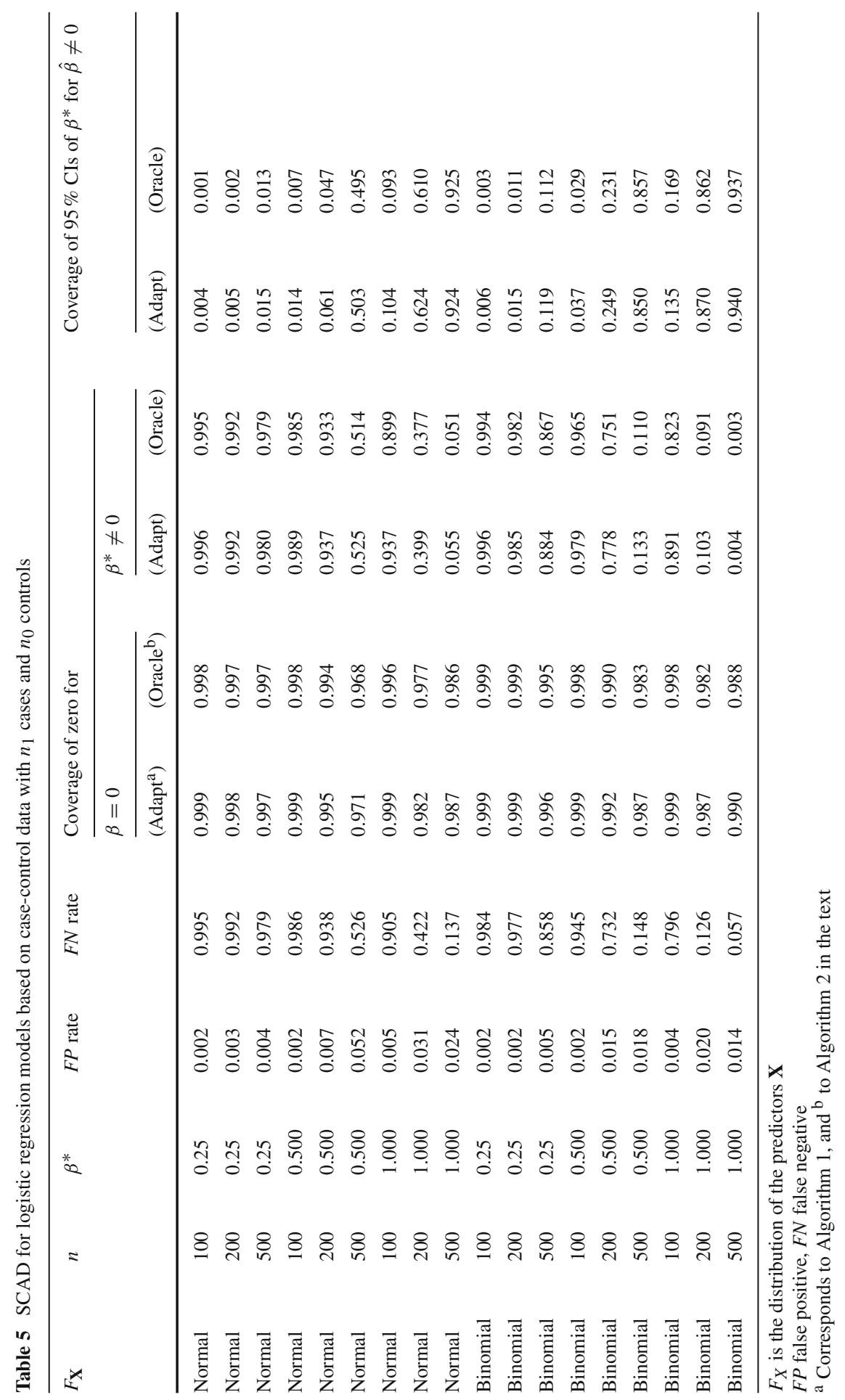




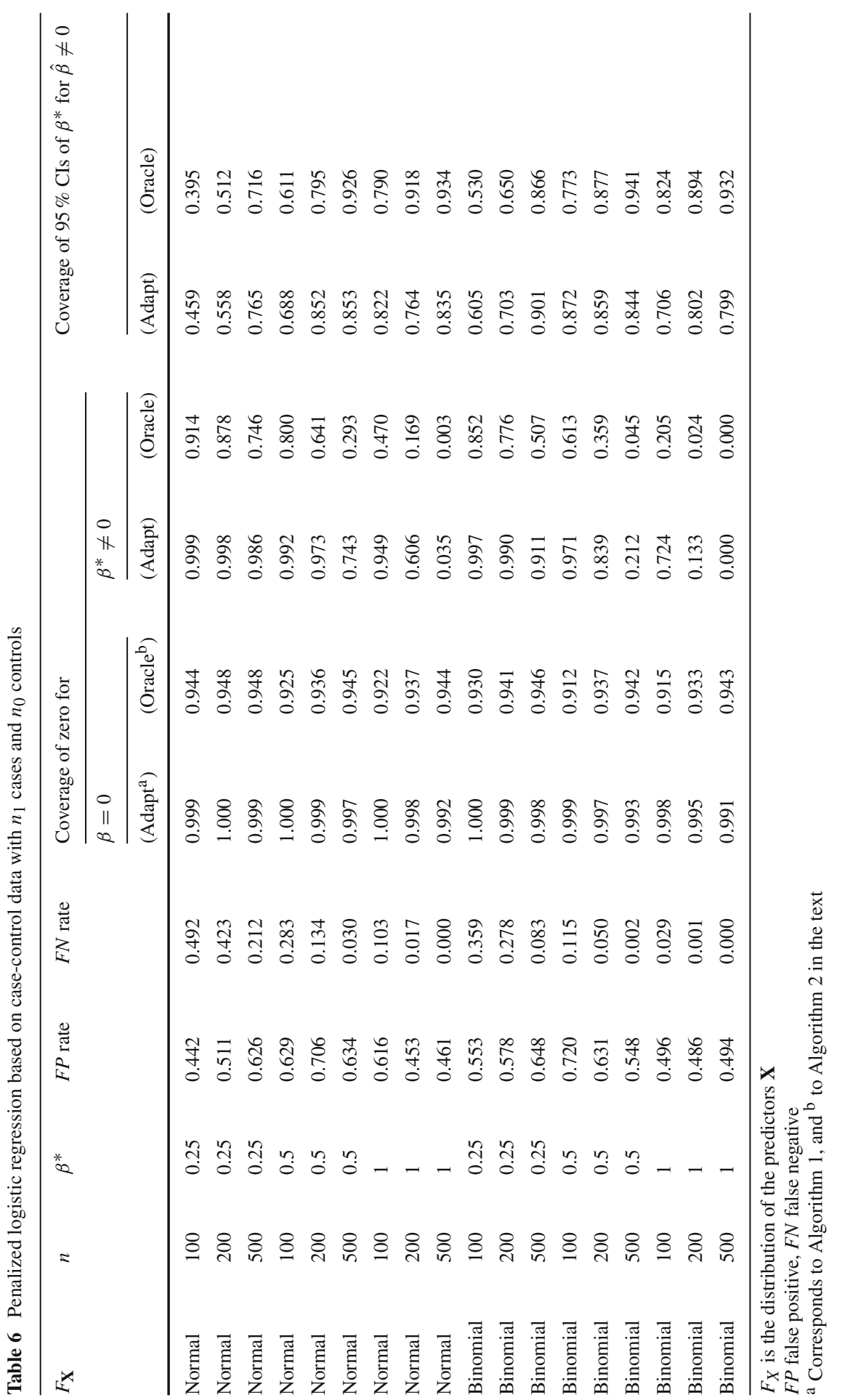




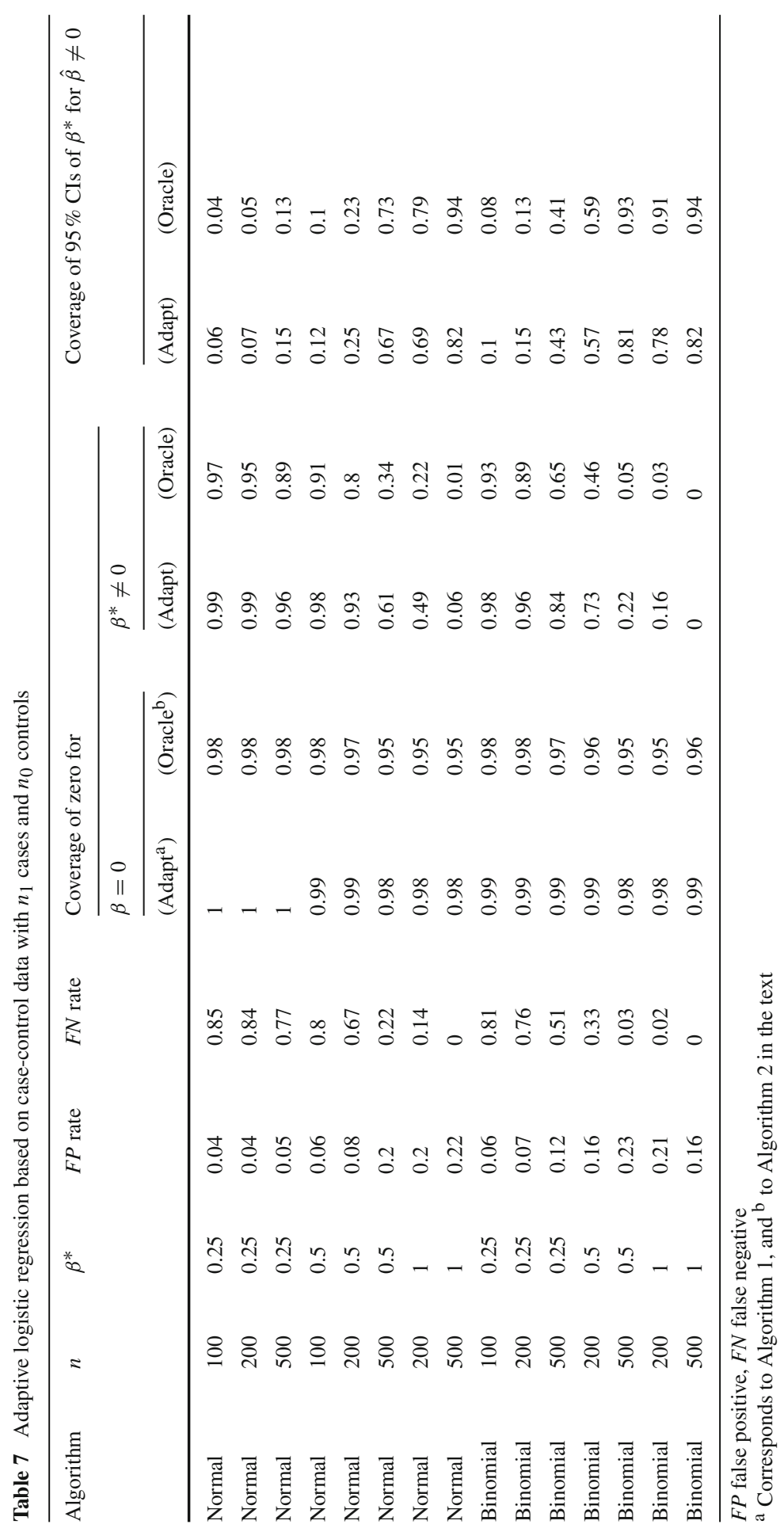


effect sizes coverage ranged from 4 to $93 \%$ for Algorithm 2 and from 6 to $82 \%$ for Algorithm 1.

\subsubsection{Summary of results for logistic regression}

LASSO and penalized logistic regression had a high FP rate and a low FN rate. SCAD had a low FP rate at the cost of having many FNs. Adaptive logistic regression had a moderate FP rate and a high FN rate. The coverage of zero for the $\beta=0$ coefficients was close to $100 \%$ for Algorithm 1, while for Algorithm 2 it was closer to $95 \%$ for all methods. The coverage of zero of the $\beta^{*}$ coefficients was close to zero for all methods with the exception of penalized logistic regression. The coverage of the true $\beta^{*}$ coefficients of the $95 \%$ CIs around $\hat{\beta} \neq 0$ was close to $95 \%$ for Algorithm 2 for large sample sizes and effect sizes for all methods with the exception of penalized logistic regression for which coverage even for $n=500$ with $\beta^{*}=1$ was around $80 \%$. It was lower for Algorithm 1.

\section{Discussion}

Penalized estimation methods deliberately introduce a bias to reduce variability of the estimates to identify outcome-associated variables, and have been typically applied to prediction. Nonetheless, penalized regression techniques are also used sometimes when the aim is inference. For example, they have been applied to molecular genetic data for both prediction, and identification of disease susceptibility genes. We therefore assessed the performance of several readily available penalized estimation methods for linear and logistic regression. We performed only a small simulation study for the setting of $p>n$ for which asymptotic results on consistent variable selection are very limited. Our main focus was on situations often encountered in practical settings, where the sample size $n$ ranges from twofold larger to tenfold larger than the number of parameters, $p$.

First we quantified the methods' ability to identify truly outcome associated predictors, i.e. to estimate the sparsity patterns of a vector $\boldsymbol{\beta}$ of regression coefficients. For linear models, penalized linear regression, elastic net, smoothly clipped absolute deviation (SCAD), least angle regression (LARS) and LASSO had a low false negative $(F N)$ predictor selection rates but false positive $(F P)$ rates above $20 \%$ for all sample and effect sizes. Partial least squares regression had few FPs but many $F N$ s. Only relaxo had low $F P$ and $F N$ rates. For logistic models, LASSO and penalized logistic regression had many FPs and few $F N$ s for all sample and effect sizes. SCAD and adaptive logistic regression had low or moderate FP rates but many FNs.

We also evaluated inference properties for the various procedures. We studied effect estimates obtained directly from penalized methods (Algorithm 1), or by refitting selected predictors with standard regression (Algorithm 2). $95 \%$ confidence interval coverage of predictors with null effects was approximately $100 \%$ for Algorithm 1 for all methods, and $95 \%$ for Algorithm 2 for large sample and effect sizes. Coverage was low only for penalized partial least squares (linear regression). For outcome- 
associated predictors, coverage was close to $95 \%$ for Algorithm 2 for large sample and effect sizes for all methods except penalized partial least squares and penalized logistic regression. Coverage was sub-nominal for Algorithm 1. In conclusion, while Algorithm 2 is preferred to Algorithm 1, estimates from Algorithm 2 are still prone to some bias arising from the selection of predictors, which affects those associated with moderate effect sizes more strongly than predictors with large effect sizes.

All procedures were somewhat sensitive to violations of the assumption of normality for the error distribution for the linear model. When we generated outcome data from a linear model where the error term $\epsilon$ followed a t-distribution with 2 degrees of freedom the $F N$ rate was higher compared to normally distributed errors for LARS, LASSO, elastic net and relaxo, while for SCAD the FP rate was much higher, and penalized partial least squares regression generally failed to give results. For outcome-associated predictors, the coverage of the $95 \%$ CIs was much below the nominal $95 \%$ for all procedures.

We addressed the problem of coverage much more extensively than previous publications (e.g. Wang and Leng 2007; Kabaila and Leeb 2006), including many popular penalized methods in our simulations, and also focused on false positive and false negative findings. We simulated practically relevant settings that reflect the number of predictors seen in many datasets, and showed that even for large sample sizes estimates are subject to undue bias and variance from the model selection procedure. Refitting attenuates the bias, but does not eliminate it in all but the cases where there is large sample size combined with estimating large effects. In these settings the residual bias not compensated for in refitting was small enough to be negligible. In all other settings where data is limited or effect sizes are small, the bias and variance are large enough to invalidate inference after model selection on those parameters, even for Algorithm 2.

When simulations were based on $p>n$, SCAD, elastic net, and penalized linear regression (the implementations we used) resulted in so many error messages that it was not meaningful to present any findings for them. For LARS and LASSO the FN rate was low and the $F P$ rate was lower than for the $p<n$ setting for moderate sample sizes but was above $74 \%$ for $n=500$ for all values of $\beta^{*}$. For relaxo, the $F P$ and $F N$ rates were similar to those seen for LARS and LASSO but low also for large $n$. The coverage of $\beta^{*}$ of the $95 \%$ CIs for $\hat{\beta} \neq 0$ coefficients was much below the nominal level for both Algorithm 1 and 2.

There is a growing literature on valid inference after model selection. E.g., Efron (2014), Wasserman and Roeder (2009) and Meinshausen et al. (2009) proposed approaches based on resampling or data splitting. Lockhart et al. (2014) derived the exact asymptotic null distribution of a test statistic for significance of variables that enter the LASSO model for general design matrices $\mathbf{X}$ and extends results to elastic net estimates. Berk et al. (2013) proposed an approach for post-selection inference ("PoSI") that is valid over all possible selected models and does not assume the linear model is correct. A better understanding of the small sample properties of some of these techniques is still needed. Nonetheless translation of the above mentioned approaches and others into statistical practice is also important to avoid misleading inference and irreproducible scientific findings. 
Acknowledgements We thank the reviewer for helpful comments and David Check for help with the figures.

Funding This study was funded by the intramural research program of the National Cancer Institute, National Institutes of Health. R. Carroll was partially supported by Grant U01-CA057030.

\section{Compliance with ethical standards}

Conflict of interest The authors declare that they have no conflict of interest.

Open Access This article is distributed under the terms of the Creative Commons Attribution 4.0 International License (http://creativecommons.org/licenses/by/4.0/), which permits unrestricted use, distribution, and reproduction in any medium, provided you give appropriate credit to the original author(s) and the source, provide a link to the Creative Commons license, and indicate if changes were made.

\section{References}

Berk R, Brown L, Buja A, Zhang K, Zhao L (2013) Valid post-selection inference. Ann Stat 41:802-837 Efron B (2014) Estimation and accuracy after model selection. J Am Stat Assoc 109:991-1007

Efron B, Hastie T, Johnstone I, Tibshirani R (2004) Least angle regression (with discussion). Ann Stat 32:407-451

Fan JQ, Li RZ (2001) Variable selection via nonconcave penalized likelihood and its oracle properties. J Am Stat Assoc 96:1348-1360

Goeman JJ (2010) L-1 penalized estimation in the Cox proportional hazards model. Biom J 52:70-84

Ihaka R, Gentlemen R (1996) R: a language for data analysis and graphics. J Comput Graph Stat 5:299-314

Kabaila P, Leeb H (2006) On the large-sample minimal coverage probability of confidence intervals after model selection. J Am Stat Assoc 101:619-629

Knight K, Fu WJ (2000) Asymptotics for Lasso-type estimators. Ann Stat 28:1356-1378

Krämer N, Boulsteix AL, Tutz G (2008) Penalized partial least squares with applications to B-spline transformations and functional data. Chemom Intell Lab 94:60-69

Leeb H (2005) The distribution of a linear predictor after model selection: conditional finite-sample distributions and asymptotic approximations. J Stat Plan Inference 134:64-89

Leeb H, Pötscher BM (2003) The finite-sample distribution of post-model-selection estimators and uniform versus nonuniform approximations. Econom Theor 19:100-142

Leeb H, Pötscher BM (2005) Model selection and inference: facts and fiction. Econom Theor 21:21-59

Leeb H, Pötscher BM (2009) On the distribution of penalized maximum likelihood estimators: the LASSO, SCAD, and thresholding. J Multivar Anal 100:2065-2082

Lockhart R, Taylor J, Tibshirani RJ, Tibshirani R (2014) A significance test for the lasso. Ann Stat 42:413468

Meier L, van de Geer S, Bühlmann P (2008) The group lasso for logistic regression. J R Stat Soc Ser B 70:53-71

Meinshausen N (2007) Relaxed Lasso. Comput Stat Data Anal 52:374-393

Meinshausen N, Meier L, Bühlmann P (2009) p-Values for high-dimensional regression. J Am Stat Assoc 104:1671-1681

Park MY, Hastie T (2008) Penalized logistic regression for detecting gene interactions. Biostatistics 9:30-50

Pötscher BM (1991) Effects of model selection on inference. Econom Theor 7:163-185

Pötscher BM, Schneider U (2009) On the distribution of the adaptive LASSO estimator. J Stat Plan Inference 139:2775-2790

Sen PK (1979) Asymptotic properties of maximum likelihood estimators based on conditional specification. Ann Stat 7:1019-1033

Tibshirani R (1996) Regression shrinkage and selection via the Lasso. J R Stat Soc Ser B 58:267-288

Wang H, Leng C (2007) Unified LASSO estimation by least squares approximation. J Am Stat Assoc 102:1039-1048

Wasserman L, Roeder K (2009) High-dimensional variable selection. Ann Stat 37:2178-2201

Zou H, Hastie T (2005) Regularization and variable selection via the elastic net. J R Stat Soc Ser B 67:301320 